\title{
Obscure Gastrointestinal Bleeding: Capsule or Balloon?
}

\author{
Bo-In Lee \\ Division of Gastroenterology, Department of Internal Medicine, Seoul St. Mary's Hospital, College of Medicine, The Catholic University of \\ Korea, Seoul, Korea
}

See "Roles of Capsule Endoscopy and Single-balloon Enteroscopy in Diagnosing Unexplained Gastrointestinal Bleeding" by Shohei Ooka, Kiyonori Kobayashi, Kana Kawagishi, et al., on page 56-60.

Obscure gastrointestinal bleeding (OGIB) is defined as overt or obscure gastrointestinal bleeding of unknown origin despite an initial endoscopic evaluation including colonoscopy and esophagogastroduodenoscopy. The majority of patients with OGIB have a bleeding source in the small intestine.

In past decades, the evaluation of the small bowel was extremely limited because of difficult intubation using conventional endoscopes. However, this limitation has been overcome by technical advances including capsule endoscopy (CE), balloon-assisted enteroscopy (BAE), and computed tomography or magnetic resonance enterography.

The main strength of $\mathrm{CE}$ is noninvasive exploration of the entire small bowel. Complete small bowel evaluation can be performed in $79 \%$ to $90 \%$ of patients, and the diagnostic yield in patients with suspected small bowel bleeding is $38 \%$ to $83 \% .{ }^{1}$

BAE is distinguished by its diagnostic and therapeutic capabilities. Double-balloon enteroscopy (DBE) was described first and single-balloon enteroscopy (SBE) followed. The basic techniques and mechanisms of SBE are similar to those of DBE. Although DBE appeared to be superior to SBE in complete small bowel evaluation, diagnostic yield, ability to treat,

Received: January 3, 2016 Accepted: January 4, 2016

Correspondence: Bo-In Lee

Division of Gastroenterology, Department of Internal Medicine, Seoul St. Mary's Hospital, College of Medicine, The Catholic University of Korea, 222 Banpo-daero, Seocho-gu, Seoul 06591, Korea

Tel: +82-2-2258-2044, Fax: +82-2-2258-2589, E-mail: gidoc4u@gmail.com

cc This is an Open Access article distributed under the terms of the Creative Commons Attribution Non-Commercial License (http://creativecommons.org/ licenses/by-nc/3.0) which permits unrestricted non-commercial use, distribution, and reproduction in any medium, provided the original work is properly cited. and complication rates are similar between DBE and SBE. ${ }^{2}$

In this issue of Clinical Endoscopy, Ooka et al. ${ }^{3}$ reported that the rate of positive findings for OGIB was significantly higher with SBE (73.6\%) than with CE (47.5\%), and SBE may be more appropriate for overt OGIB. However, caution is required in the interpretation of the results of this report. CE was performed before SBE in $28.5 \%$ of the SBE group in this study. CE is useful for screening before performing BAE in patients with OGIB, and CE before BAE is known to increase the diagnostic and therapeutic yields. ${ }^{4}$ In this study, CE might have improved the diagnostic yield in the SBE group, although CE was performed in a part of the SBE group.

$\mathrm{CE}$ can also guide the insertion route of BAE. An oral approach is usually preferred when a lesion is located within the proximal two-thirds of the small bowel. In this issue of Clinical Endoscopy, it did not appear that a bidirectional approach was tried, because SBE was performed in 91 patients and the oral and anal approaches in 43 and 48, respectively. When an oral or anal approach fails to detect significant findings or reach the targeted lesion, the other route can be tried in practice. A 73.6\% diagnostic yield by SBE in patients with OGIB is relatively surprising, even though only one route was tried in all cases.

Diagnostic yield of CE and BAE for patients with overt bleeding is higher than that for patients with occult bleeding, and the diagnostic yield for patients with ongoing overt bleeding is higher than that for patients with previous overt bleeding. In Ooka's study, ${ }^{3}$ the percentage of patients with overt bleeding was higher in the SBE group than in the CE group ( $80.8 \%$ vs. $58.4 \%$ ), and ongoing overt bleeding was also more 
frequent $(100.0 \%$ vs. $90.9 \%)$ in the SBE group. The results might have changed if the authors performed multivariate analyses according to the amount and timing of bleeding.

Actually, $\mathrm{CE}$ and $\mathrm{BAE}$ are not competitive, but complementary. According to the American College of Gastroenterology guidelines, CE should be considered a first-line procedure for suspected small bowel bleeding, and BAE should be attempted if a small bowel lesion is strongly suspected based on clinical presentation and abnormal CE results. ${ }^{5}$ However, the results of Ooka's study do not seem to be sufficient to change current diagnostic guidelines for small bowel bleeding.

\section{Conflicts of Interest}

The author has no financial conflicts of interest.

\section{REFERENCES}

1. Rondonotti E, Villa F, Mulder CJ, Jacobs MA, de Franchis R. Small bowel capsule endoscopy in 2007: indications, risks and limitations. World J Gastroenterol 2007;13:6140-6149.

2. Wadhwa V, Sethi S, Tewani S, et al. A meta-analysis on efficacy and safety: single-balloon vs. double-balloon enteroscopy. Gastroenterol Rep (Oxf) 2015;3:148-155.

3. Ooka S, Kobayashi K, Kawagishi K, et al. Roles of capsule endoscopy and single-balloon enteroscopy in diagnosing unexplained gastrointestinal bleeding. Clin Endosc 2016;49:56-60.

4. Fry LC, Neumann H, Jovanovic I, Malfertheiner P, Mönkemüller K. Capsule endoscopy increases the diagnostic yield of double balloon enteroscopy in patients being investigated for obscure gastrointestinal bleeding. Arch Gastroenterohepatol 2012;29:9-14.

5. Gerson LB, Fidler JL, Cave DR, Leighton JA. ACG clinical guideline: diagnosis and management of small bowel bleeding. Am J Gastroenterol 2015;110:1265-1287. 